\title{
MicroRNA-124 represses wound healing by targeting SERP1 and inhibiting the Wnt/B-catenin pathway
}

\author{
Guohui Zhang ${ }^{1, B, D, F}$, , Kunxiu Song ${ }^{2, C, D, F}$, Hongshan Yan ${ }^{3, A, D-F}$ \\ ${ }^{1}$ Department of Burn and Plastic Surgery, Binzhou Medical University Hospital, China \\ ${ }^{2}$ Department of Hand and Microsurgery, Binzhou Medical University Hospital, China \\ ${ }^{3}$ Jiangsu Liyang Zhimeixing Medical Cosmetic Clinic, China \\ A - research concept and design; B - collection and/or assembly of data; C - data analysis and interpretation; \\ $D$ - writing the article; $E$ - critical revision of the article; $F$ - final approval of the article
}

\section{Address for correspondence \\ Hongshan Yan}

E-mail: yanhongshan123@126.com

Funding sources

None declared

Conflict of interest

None declared

Received on March 9, 2017

Reviewed on June 7, 2017

Accepted on August 9, 2018

Published online on February 8, 2019

Cite as

Zhang G, Song K, Yan H. MicroRNA-124 represses wound healing by targeting SERP1 and inhibiting the Wnt/ $\beta$-catenin pathway. Adv Clin Exp Med. 2019;28(6):711-718. doi:10.17219/acem/94163

DOI

10.17219/acem/94163

\section{Copyright}

Copyright by Author(s)

This is an article distributed under the terms of the

Creative Commons Attribution Non-Commercial License

(http://creativecommons.org/licenses/by-nc-nd/4.0/)

\begin{abstract}
Background. Wound healing is a complex process which restores cellular structures and tissue layers after their destruction. Accumulating evidence has proven that microRNAs (miRs) are involved in wound healing.

Objectives. The aim of the study was to research the role of miR-124 in wound healing.

Material and methods. Keratinocytes were respectively transfected with miR-124 mimic, scrambled miRNA (a negative control of miR-124 mimic: mimic NC), antisense oligonucleotides against miR-124 (ASOmiR-124), or a negative control of ASO-miR-124 (ASO-NC), and then cell viability, colony formation, cell cycle, expression of cell cycle-associated proteins, and collagen content were all evaluated. The target gene of miR-124 was predicted using TargetScan and verified with luciferase assay. Subsequently, the effects of target gene overexpression on cell viability, colony formation and collagen synthesis were all evaluated. Finally, the expression levels of key kinases in the Wnt/B-catenin pathway were detected using western blot analysis.

Results. Cell viability, colony formation, expression levels of cell cycle-associated proteins, and collagen content were all significantly reduced by miR-124 overexpression. As predicted using bioinformatics and validated with luciferase assay, stress-associated endoplasmic reticulum protein 1 (SERP1) is a target gene of miR-124. Meanwhile, the miR-124 mimic-induced decrease in cell viability, colony formation and collagen synthesis was reversed by SERP1 overexpression. Furthermore, the miR-124 mimic obviously upregulated

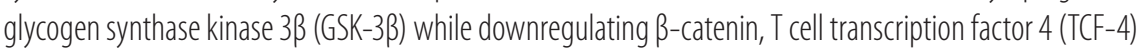
and leukemia enhancer factor 1 (LEF-1). Additionally, all the effects of ASO-miR-124 were the opposite of those of the miR-124 mimic.
\end{abstract}

Conclusions. We found that miR-124 inhibited keratinocyte proliferation, collagen biosynthesis and activation of Wnt/B-catenin by targeting SERP1.

Key words: wound healing, collagen biosynthesis, Wnt/B-catenin pathway, miR-124, keratinocyte proliferation 
Wound healing is a complex biological process in which cellular structures and tissue layers are restored after their destruction. ${ }^{1}$ Approximately $1-1.5 \%$ of the population in the developed world suffers from chronic wounds and the costs of their treatment account for $2-4 \%$ of the healthcare budget. ${ }^{2}$ The process of wound healing consists of 4 distinct but overlapping phases: hemostasis, inflammation, proliferation, and tissue remodeling. ${ }^{3}$ These 4 phases must be orchestrated in a precise and regulated manner; otherwise, wound healing can be delayed or even prevented. ${ }^{4}$ Scars are usually formed by excessive non-functioning disorganized deposition of extracellular matrix (ECM) components during normal wound healing. ${ }^{5}$ However, keloids and hypertrophic scars, which are formed by prolonged and aberrant ECM accumulation during burns, surgery and wounds, can cause functional disability, cosmetic deformities, psychological stress, and a huge socioeconomic burden. ${ }^{6}$ Due to the deficiencies of current therapeutic approaches, it is urgent to explore novel targets that could promote wound healing and help avoid keloids or hypertrophic scars.

MicroRNAs (miRs) are a category of highly conserved small non-coding RNAs 18-22 nt in length. ${ }^{7}$ Recently, increasing numbers of miRs have been reported as participating in wound healing through recognizing and binding complementarily to the 3'-untranslated region (3'UTR) of target genes, resulting in degradation of mRNA or repression of translation. ${ }^{8}$ For example, miR-27b accelerates wound healing in type 2 diabetes mellitus. ${ }^{9}$ Another report showed that miR-21 regulates wound contraction and collagen deposition by targeting various aspects of the healing process. ${ }^{10}$ In addition, miR-1908 plays a positive role in scar formation by suppressing Skimediated inflammation in vitro and in vivo. ${ }^{11}$ The functional role of miR-124 has been widely investigated in various cancers, including ovarian cancer, ${ }^{12}$ non-small-cell lung cancer, ${ }^{13}$ esophageal cancer, ${ }^{14}$ and breast cancer. ${ }^{15}$ However, the specific role of miR-124 in wound healing remains unclear.

The process of wound healing involves various types of cells, including inflammatory cells, keratinocyte, fibroblasts, and endothelial cells. ${ }^{4}$ As the major cell type in the epidermal layer, keratinocytes migrate to cover the lesion and restore the barrier function of the skin during wound healing. When the wound area is totally covered, the keratinocytes are differentiated to a basal phenotype by contact inhibition. ${ }^{16}$ Considering the essential roles of keratinocyte in wound healing, we chose keratinocytes to explore the effects of miR-124 on wound healing and the underlying mechanisms. Furthermore, the possible target gene of miR-124 was also investigated.

\section{Material and methods}

\section{Cell culture and transfection}

Primary human epidermal keratinocytes were purchased from the American Type Culture Collection (ATCC,
Manassas, USA). The keratinocytes were maintained in Dermal Cell Basal Medium (ATCC) supplemented with a Keratinocyte Growth Kit (ATCC) at $37^{\circ} \mathrm{C}$ with $5 \%$ $\mathrm{CO}_{2}$. To obtain non-physiological expression of miR-124, the keratinocytes were transfected with miR-124 mimic, scrambled miRNA (a negative control of miR-124 mimic: mimic NC), antisense oligonucleotides against miR-124 (ASO-miR-124), or a negative control of ASO-miR-124 (ASO-NC) (all from GenePharma, Shanghai, China). To overexpress stress-associated endoplasmic reticulum protein 1 (SERP1), complete SERP1 sequences were cloned into a pcDNA3.1/Zeo vector (Invitrogen, San Diego, USA) to generate pcDNA3.1/SERP1 (pc-SERP1); the recombined plasmid was then transfected into keratinocytes. The keratinocytes were seeded with a density of $1 \times 10^{5}$ cells/well and were maintained to $30-50 \%$ confluence. Cell transfection was then performed using Lipofectamine 2000 transfection reagent (Invitrogen) according to manufacturer's instructions. The keratinocytes were divided into 4 groups (mimic NC, miR-124 mimic, ASO-miR-124, and ASO-NC) or 3 groups (mimic NC, miR-124 mimic and miR-124 mimic + pc-SERP1) through cell transfection. Each group included 3 replicate wells.

\section{Cell viability and cell cycle}

Cell viability was measured with MTS assay using the CellTiter 96 AQueous One Solution Cell Proliferation Assay (Promega Corp., Madison, USA). Briefly, keratinocytes were seeded into a 96-well plate. At $24 \mathrm{~h}, 48 \mathrm{~h}$ and $72 \mathrm{~h}$ after transfection, $20 \mu \mathrm{L}$ of AQueous One Solution Reagent (Promega Corp.) was added to each well and the keratinocytes were incubated at $37^{\circ} \mathrm{C}$ for additional $4 \mathrm{~h}$. The absorbance was detected with a microplate reader (Bio-Rad Laboratories Inc., Hercules, USA) at $490 \mathrm{~nm}$. To determine the cell cycle, keratinocytes were collected at $48 \mathrm{~h}$ posttransfection and washed in phosphate-buffered saline (PBS; Beyotime, Shanghai, China). The keratinocytes were then fixed in cold $70 \%$ ethanol at $4^{\circ} \mathrm{C}$ overnight. The fixed keratinocytes were washed in PBS until the ethanol was thoroughly decanted. Afterwards, the keratinocytes were incubated in propidium iodide solution (PI, $50 \mu \mathrm{g} / \mathrm{mL}$; Sigma-Aldrich, St. Louis, USA) at $37^{\circ} \mathrm{C}$ for $30 \mathrm{~min}$ in the dark. The fluorescence intensity of the stained keratinocytes was detected on a FACSCalibur flow cytometer (BD Biosciences, San Jose, USA) and the data was analyzed using FlowJo software (Tree Star Inc., San Carlos, USA).

\section{Colony formation assay}

Twenty-four hours after transfection keratinocytes were plated into a 12-well plate at a density of 300 cells/well. The keratinocytes were then cultured at $37^{\circ} \mathrm{C}$ for 14 days; the culture medium was changed every 3 days. After being stained with $2 \%$ crystal violet, the colonies that included more than 50 cells were counted. 


\section{RNA isolation and qRT-PCR}

The total RNA of transfected keratinocytes was extracted with TRIzol reagent (Invitrogen) according to the manufacturer's instructions, and reverse transcription of $1 \mu \mathrm{g}$ RNA was performed using an mRNA Selective PCR kit (Takara, Dalian, China). Using cDNA as a template (50 ng), quantitative real-time polymerase chain reaction (qRTPCR) was performed by means of the Power SYBR Green PCR Master Mix with the 7500 Fast Real-Time PCR system (Applied Biosystems, Foster City, USA). The primers were designed and synthesized by Sangon (Shanghai, China). The relative expression fold was calculated according to the $2^{-\triangle \Delta C t}$ method. ${ }^{17} G A P D H$ acted as the housekeeping gene.

\section{Protein extraction and western blot analysis}

Transfected keratinocytes were lysed in ice-cold RIPA buffer (Beyotime) containing a protease inhibitor cocktail (Roche Diagnostics GmbH, Mannheim, Germany). After quantification with a bicinchoninic acid (BCA) assay kit (Pierce Biotechnology, Rockford, USA), lysates containing approx. $30 \mu \mathrm{g}$ of proteins were separated using sodium dodecyl sulfate polyacrylamide gel electrophoresis (SDSPAGE), and the proteins in the SDS-PAGE gels were further transferred onto polyvinylidene fluoride (PVDF) membranes (EMD Millipore, Billerica, USA). After blocking and incubation with primary antibodies against cyclin B1 (ab32053), cyclin D1 (ab137875), cyclin-dependent kinase 2 (CDK2, ab32147), collagen I (ab34710), SERP1 (ab130974), $\beta$-catenin (ab6302), glycogen synthase kinase $3 \beta$ (GSK-3 $\beta$, ab131356), T-cell transcription factor 4 (TCF-4, ab185736), leukemia enhancer factor 1 (LEF-1, ab52017), or GAPDH (ab128915) (all from Abcam, Cambridge, UK), the blots were detected using appropriate secondary horseradish peroxidase-conjugated secondary antibodies (Abcam) and visualized using enhanced chemiluminescence (Pierce Biotechnology). The intensity of the bands was detected using Image Lab software (Bio-Rad Laboratories Inc.) and quantified using Image J software (National Institutes of Health, Bethesda, USA).

\section{Collagen assay}

The quantity of fibrillar collagens (type I to V) was assayed using the Sircol Soluble Collagen Assay Kit (Biocolor Ltd., Carrickfergus, UK) according to the manufacturer's protocol. Briefly, the cell culture medium was collected at $48 \mathrm{~h}$ post-transfection and mixed with Sircol dye reagent, which specially binds to the Gly-X-Y fragment in the helical structure. The serum concentration of the culture medium must be no more than $5 \%$ at this point. After incubation for $30 \mathrm{~min}$ at room temperature, the mixture was centrifuged and the precipitate was mixed with ice-cold acidsalt washing reagent, followed by another centrifugation.
The precipitate was dissolved in Alka reagent for approx. $5 \mathrm{~min}$ and the absorbance was detected under $555 \mathrm{~nm}$.

\section{Luciferase reporter assay}

Putative target genes of miR-124 were predicted using a TargetScan algorithm. A wild-type (WT) 3'UTR fragment containing a putative binding site for miR-124 and mutant (Mut) 3'UTR with site-mutagenesis at the binding site were inserted into a pmirGLO Vector (Promega Corp.). The mutant was constructed using the Directed Mutagenesis System (Invitrogen) with the sequence of SERP1 at the position between 188 and 194 as well as the position between 1645 and 1652. For the luciferase reporter assays, keratinocytes were seeded in a 24 -well plate $\left(1 \times 10^{5}\right.$ cells/well $)$ and maintained for $24 \mathrm{~h}$ before transfection. The 3'UTR reporter plasmids (WT or Mut) and miR-124 mimic, mimic NC, ASO-miR-124, or ASO-NC were co-transfected into the keratinocytes using Lipofectamine 2000 reagent (Invitrogen). The transfected keratinocytes were harvested $24 \mathrm{~h}$ later and assayed using the Dual-Luciferase Reporter Assay System (Promega Corp.) according to the manufacturer's protocol. Firefly luciferase activity was normalized to renilla luciferase activity for each well.

\section{Statistical analysis}

All the experiments were repeated 3 times. The results are presented as means \pm standard deviation (SD). The statistical analysis was performed using GraphPad Prism 5 software (GraphPad Software Inc., San Diego, USA). The p-values were calculated using the unpaired twotailed t-test or one-way analysis of variance (ANOVA) with Bonferroni's correction. A p-value less than 0.05 (marked by *) was considered statistically significant.

\section{Results}

\section{MicroRNA-124 is non-physiologically expressed in keratinocytes}

Mimic NC, miR-124 mimic, ASO-NC, and ASO-miR-124 were transfected into keratinocytes. The results in Fig. 1 showed that the miR-124 level was significantly upregulated after transfection with miR-124 mimic compared to the mimic NC group ( $\mathrm{p}<0.01$ ), but was markedly downregulated after transfection with ASO-miR-124 compared to the ASO-NC group ( $\mathrm{p}<0.01$ ). This data indicates that miR-124 was non-physiologically expressed in keratinocytes after cell transfection.

\section{MicroRNA-124 inhibits keratinocyte proliferation}

To investigate the effects of miR-124 on keratinocytes, cell proliferation, cell viability, colony formation, the cell 


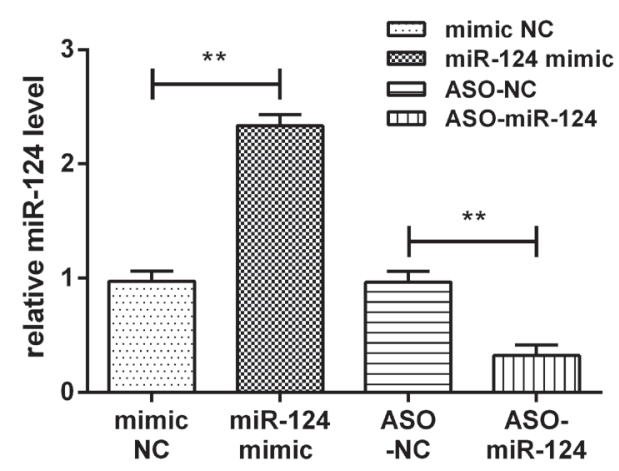

Fig. 1. MicroRNA miR-124 is non-physiologically expressed in keratinocytes after cell transfection. Keratinocytes were transfected with miR-124 mimic, mimic NC, ASO-miR-124, or ASO-NC. The level of miR-124 was measured using quantitative real-time polymerase chain reaction (qRT-PCR). The data presented is the means of at least 3 independent experiments. Error bars indicate the standard deviation (SD)

** $p<0.01$. cycle, and expressions of cell cycle-associated proteins were all assessed in transfected keratinocytes. In Fig. 2A, cell viability was markedly reduced by miR-124 mimic compared with the mimic NC group at $24 \mathrm{~h}(\mathrm{p}<0.05), 48 \mathrm{~h}(\mathrm{p}<0.01)$ and $72 \mathrm{~h}(\mathrm{p}<0.01)$ after transfection. Meanwhile, the number of colonies formed in keratinocytes overexpressing miR-124 was obviously lower than in the mimic NC group ( $\mathrm{p}<0.05$, Fig. 2B). The fluorescence-activated cell sorting (FACS) results in Fig. 2C show that miR-124 overexpression reduced the number of keratinocytes in the $S$ phase, while it increased the number in $G_{0} / G_{1}$ phase, accompanied by nearly unchanged numbers in the $G_{2} / M$ phase when compared with the mimic NC group. In addition, mRNA and protein expression levels of cyclinB1, cyclinD1 and CDK2 were all significantly decreased by miR-124 overexpression in comparison with the mimic NC group $(\mathrm{p}<0.05$ or $\mathrm{p}<0.01$, Fig. 2D-E). Also, the effects of ASO-miR-124 on keratinocytes were the opposite to those of miR-124
A

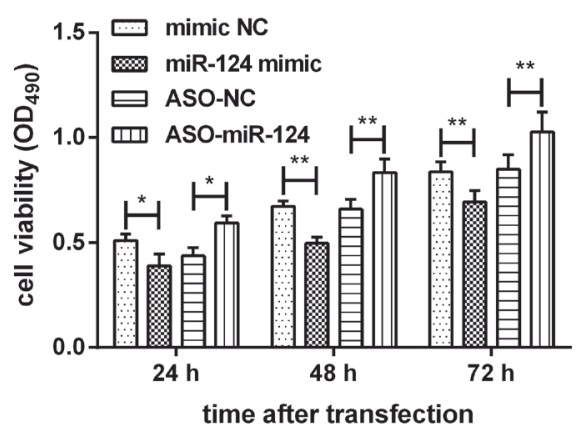

C

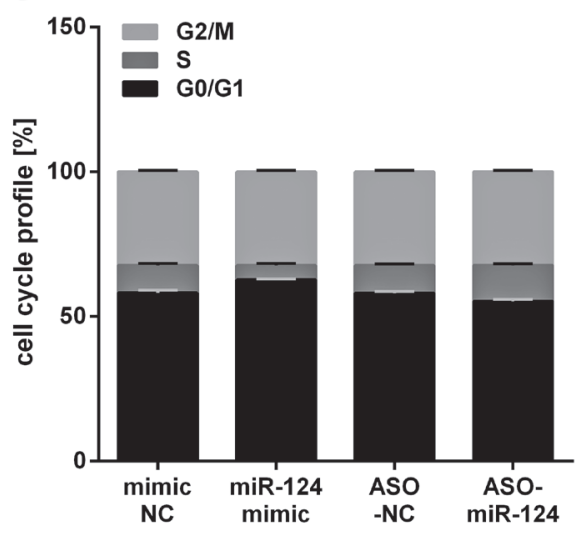

E

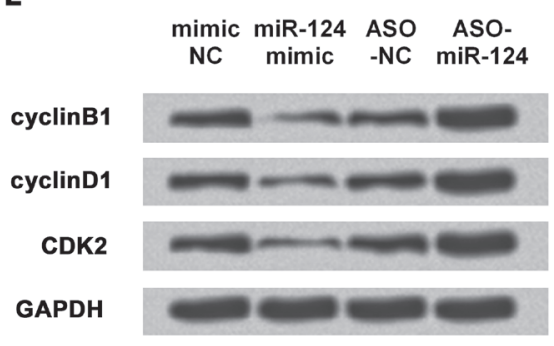

mimic NC

miR-124 mimic
B

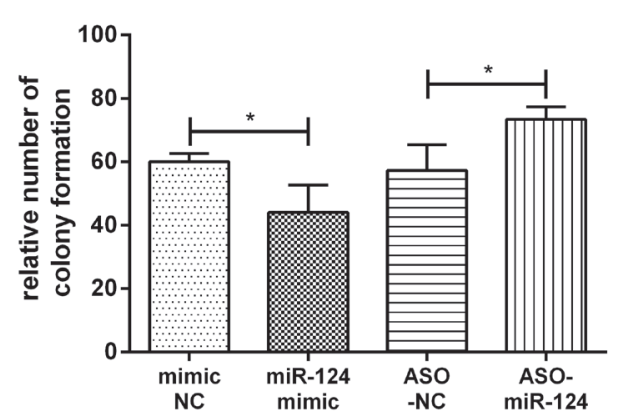

D
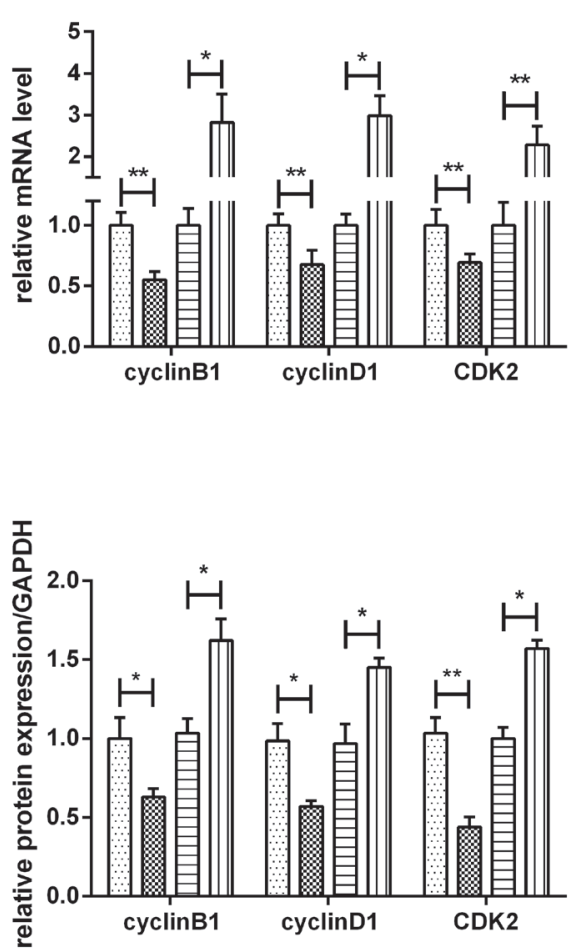

ASO-NC
Fig. 2. MicroRNA miR-124 inhibits keratinocyte proliferation. Keratinocytes were transfected with miR-124 mimic, mimic NC, ASO-miR-124, or ASO-NC. A) cell viability by MTS assay; B) colony formation rate;

C) cell cycle by fluorescenceactivated cell sorting (FACS) assay; D) mRNA expression levels of cell cycle-associated proteins measured using quantitative real-time polymerase chain reaction (qRT-PCR); E) protein expression levels of cell cycle-associated proteins measured using western blot analysis. The data presented is the means of at least 3 independent experiments. Error bars indicate the standard deviation (SD) ${ }^{*} p<0.05$; * $p<0.01$; CDK2 - cyclindependent kinase 2 . 


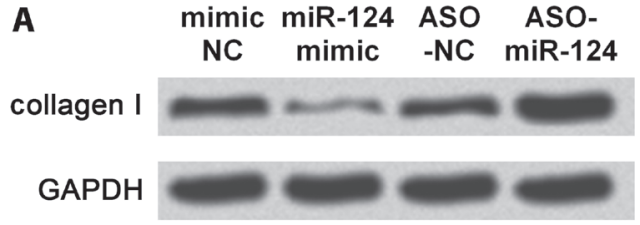

B

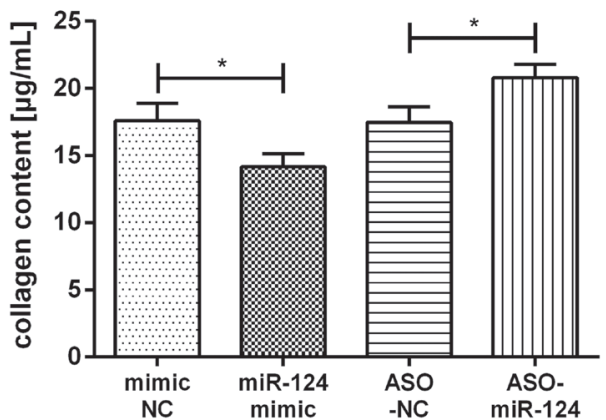

Fig. 3. MicroRNA miR-124 inhibits collagen biosynthesis. Keratinocytes were transfected with miR-124 mimic, mimic NC, ASO-miR-124, or ASO-NC. A) expression of collagen I measured using western blot analysis; B) collagen content in the culture medium measured using collagen assay. The data presented is the means of at least 3 independent experiments. Error bars indicate the standard deviation (SD)

${ }^{*} p<0.05$ mimic. Taking all this together, we concluded that miR-124 inhibited the cell proliferation of keratinocytes.

\section{MicroRNA-124 inhibits collagen biosynthesis}

To assess the effects of miR-124 on collagen biosynthesis, intracellular collagen and collagen in a culture medium were both evaluated. In Fig. 3A, intracellular collagen I was markedly downregulated by miR-124 mimic, while it was upregulated by ASO-miR-124. Likewise, the content of collagens in the culture medium of keratinocytes overexpressing miR-124 was lower than in the mimic NC group ( $p<0.05$, Fig. 3B). The effect of ASO-miR-124 was just the opposite. This demonstrates that miR-124 inhibited collagen biosynthesis in keratinocytes.

\section{SERP1 is a target gene of miR-124}

After screening with a TargetScan algorithm, SERP1 was identified as a putative target gene of miR-124. The complement sequence is shown in Fig. 4A. To identify whether
A

SERP1

(188-194) (1645-1652)

3'UTR WT 5'...ACCACAgugccuuG...AGgugccuuA...3' \|\|\|\|$\quad\|\|\|\|$

hsa-miR-124 3'...GCGcacggaa...GCGcacggaaU...5'

3'UTR Mut 5'...ACCACAacaaaccG...AGaccaaggA...3'
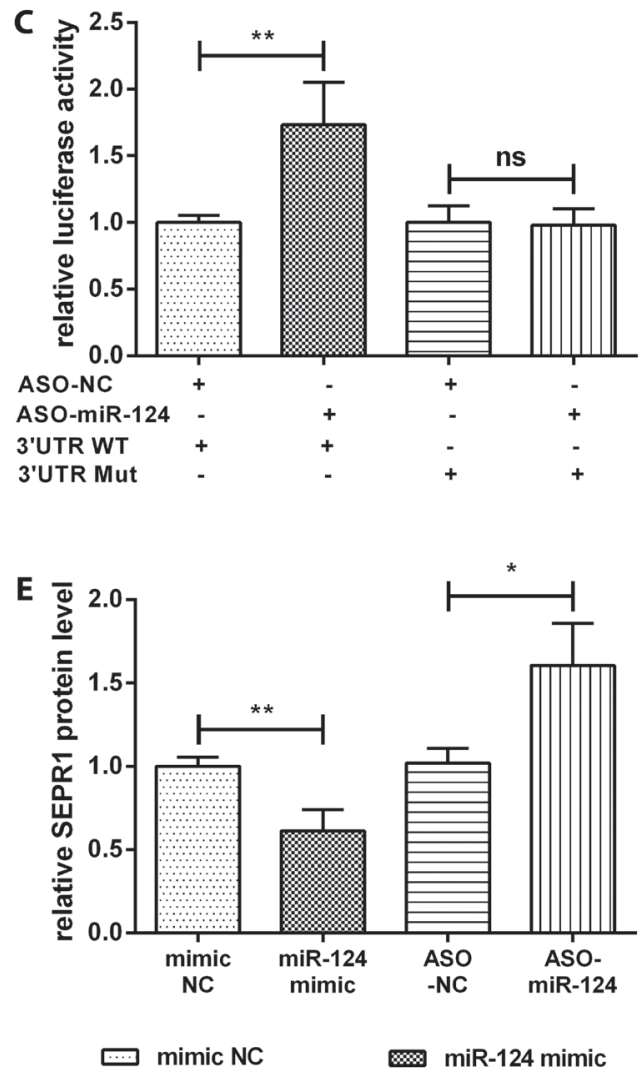

B

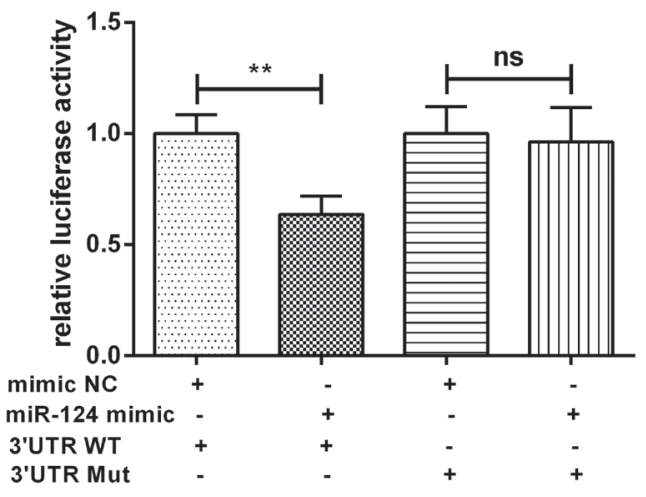

D

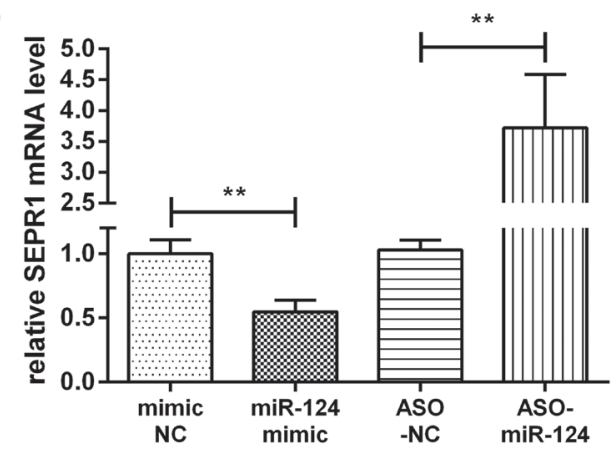

$\mathbf{F}$

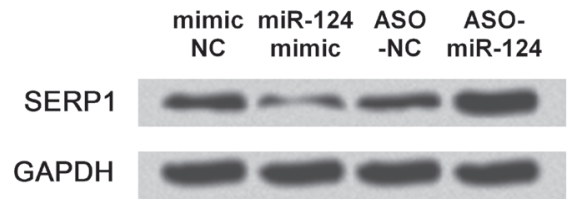

$\square$ ASO-NC

ASO-miR-124
Fig. 4. SERP1 is a target gene of microRNA (miR)-124.

A) sequence complementarity between miR-124 and the seed region in the SERP1 3'-untranslated region (3'UTR) reporter plasmids (WT or Mut); short vertical lines indicate complementary nucleotides; B) relative luciferase activity in keratinocytes after co-transfection with miR-124 mimic or mimic NC and SERP1 3'UTR plasmid (WT or Mut);

C) relative luciferase activity in keratinocytes after co-transfection with ASO-miR-124 or ASO-NC and SERP1 3'UTR plasmid (WT or Mut):

D) relative SERP1 mRNA; E-F) protein expression levels after transfection with mimic NC, miR-124 mimics, ASO-NC, or ASO-miR-124. The data presented is the means of at least 3 independent experiments. Error bars indicate the standard deviation (SD)

ns: $p>0.05 ;{ }^{*} p<0.05$ ** $p<0.01$; WT - wild-type; Mut - mutant. 
A
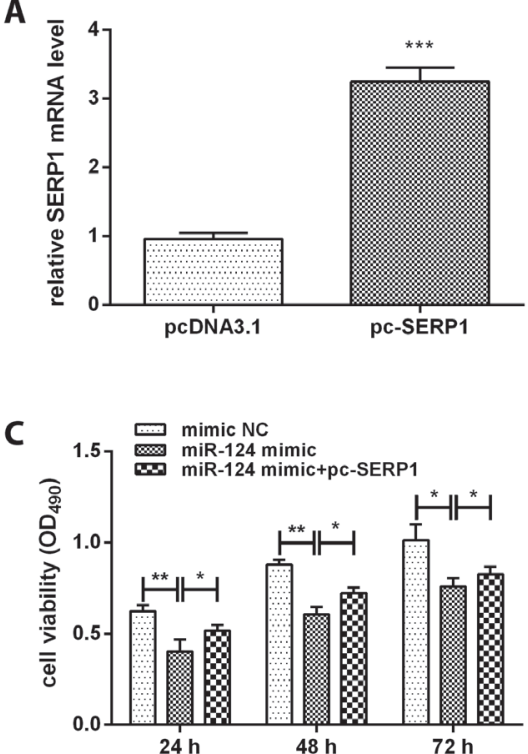

B

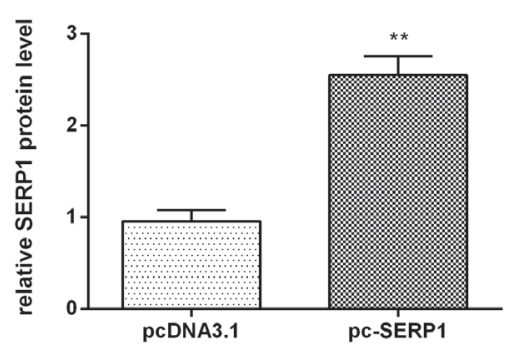

D

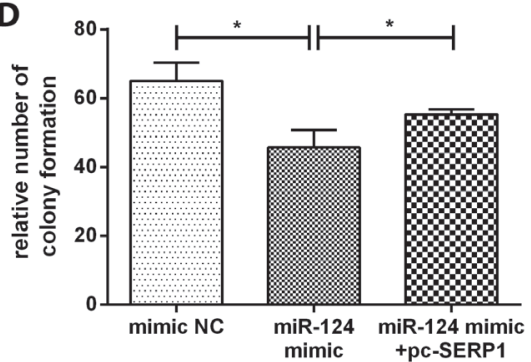

pc-DNA3.1 Pc-SERP1

SERP1

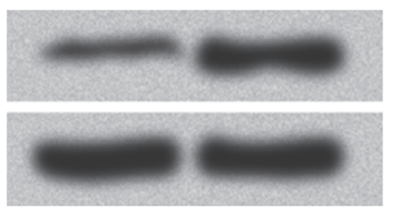

GAPDH

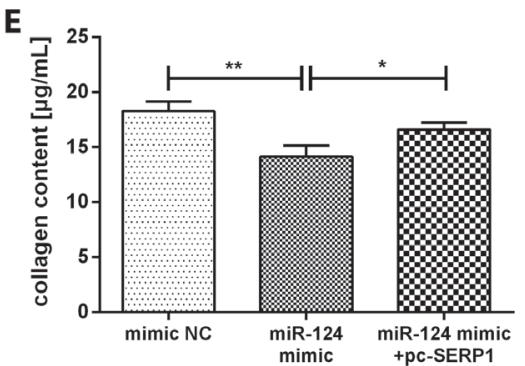

Fig. 5. SERP1 reverses the inhibitory effects of miR-124 on keratinocytes. Keratinocytes were transfected with pcDNA3.1 or pcDNA3.1/SERP1 (pc-SERP1), followed by (A) quantitative real-time polymerase chain reaction (qRT-PCR) assessing the mRNA levels of SERP1 and (B) western blot analysis for assessing the protein levels of SERP1. Keratinocytes were transfected with mimic NC, miR-124 mimic alone or accompanied by pc-SERP1; C) cell viability measured using MTS assay; D) colony formation rate; E) collagen content in the culture medium measured using collagen assay. The data presented is the means of at least 3 independent experiments. Error bars indicate the standard deviation (SD)

${ }^{*} p<0.05 ;{ }^{* *} p<0.01 ;{ }^{* * *} p<0.001$.

miR-124 directly targets $S E R P 1$, luciferase activity was assessed after co-transfection. As shown in Fig. 4B, miR-124 mimic markedly decreased the luciferase activity of keratinocytes transfected with plasmid containing WT 3'UTR but not Mut 3'UTR ( $<$ 0.01). Meanwhile, ASO-miR-124 significantly increased the luciferase activity of keratinocytes transfected with plasmid containing WT 3'UTR but not Mut 3'UTR reporter ( $\mathrm{p}<0.01$ ), as shown in Fig. $4 \mathrm{C}$. In Fig. 4D-F, the mRNA and protein expression levels of SERP1 were both reduced in keratinocytes overexpressing miR-124 compared with the mimic NC group ( $\mathrm{p}<0.01)$. At the same time, the expression levels of SERP1 were elevated in keratinocytes with ASO-miR-124 compared with the ASO-NC group ( $\mathrm{p}<0.05$ or $\mathrm{p}<0.01$ ). All these results suggest that SERP1 is a target gene of miR-124 and its expression was negatively regulated by miR-124.

\section{SERP1 overexpression rescues inhibitory effects of miR-124 on keratinocytes}

After pcDNA3.1 or pc-SERP1 was transfected into keratinocytes, mRNA and protein expression levels of SERP1 were assessed. As shown in Fig. 5A-B, both mRNA and protein expression levels of SERP1 were significantly upregulated after transfection with pc-SERP1 compared to the pcDNA3.1 groups ( $\mathrm{p}<0.01$ or $\mathrm{p}<0.001$ ). Cell viability, which was decreased by miR-124 overexpression, was obviously increased by SERP1 overexpression when compared to the miR-124 mimic group at $24 \mathrm{~h}, 48 \mathrm{~h}$ and $72 \mathrm{~h}$ (all p $<0.05$, Fig. 5C) after transfection. Analogically, the colony formation rate and collagen content, which were reduced by miR-124 overexpression, were both enhanced by SERP1 overexpression when compared to the corresponding miR-124 mimic group ( $<<0.05$, Fig. 5D-E). These results imply that $S E R P 1$ overexpression could reverse the effects of miR-124 overexpression on keratinocytes.

\section{MicroRNA-124 represses activation of the Wnt/ $\beta$-catenin pathway}

To reveal the underlying mechanisms of miR-124 modulations, the expression of key kinases involved in the Wnt/ $\beta$-catenin pathway was assessed with western blot analysis in transfected keratinocytes. The results presented in Fig. 6A showed that miR-124 mimic obviously downregulated $\beta$-catenin but upregulated GSK-3 $\beta$. Further results suggested that TCF-4 and LEF-1 expression were both downregulated by miR-124 mimic (Fig. 6B). Not surprisingly, the effects of ASO-miR-124 on the expressions of these kinases were just the opposite. All these results indicate that miR-124 repressed activation of the Wnt/ $\beta$ catenin pathway in keratinocytes.

\section{Discussion}

Considering the deficiencies of current therapeutic treatments for the effective reduction of skin scar formation, it is an unmet challenge for clinicians to explore novel therapeutic targets. In this study, we found that miR-124 

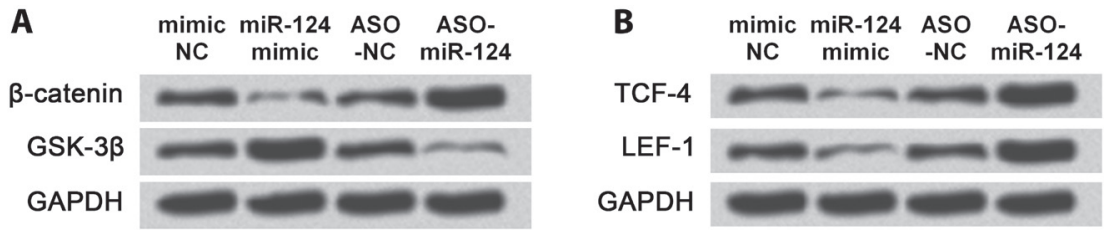

Fig. 6. MicroRNA miR-124 represses activation of the Wnt/ $\beta$-catenin signaling pathway. Keratinocytes were transfected with miR-124 mimic, mimic NC, ASO-miR-124, or ASO-NC. A) protein expressions of $\beta$-catenin and glycogen synthase kinase $3 \beta$ (GSK-3 $\beta$ ) measured using western blot analysis;

B) protein expressions of TCF-4 and LEF-1 measured using western blot analysis significantly reduced cell viability, lowered the colony formation rate and downregulated expressions of cell cycle-associated proteins as well as collagens. Additionally, miR-124 overexpression induced $G_{0} / G_{1}$ phase arrest in keratinocytes. The effects of miR-124 silence on keratinocytes were just the opposite. Afterwards, using the online TargetScan software, SERP1 was hypothesized to be a target gene of miR-124, and the subsequent luciferase assay verified the hypothesis. Meanwhile, SERP1 overexpression could reverse the effects of miR-124 overexpression, also validating this hypothesis. The final western blot analysis suggested that miR-124 deactivated the Wnt/ $\beta$-catenin signaling pathway.

During the course of wound healing, re-epithelialization is pivotal to optimal wound closure due to its role in wound contraction. ${ }^{18}$ The process of re-epithelialization is partially mediated by keratinocyte proliferation. ${ }^{19}$ At the wound margin, keratinocytes begin to proliferate behind the migrating cells and thereby feed the migrating sheets. ${ }^{20}$ Thus, cell proliferation of keratinocytes plays an essential role in wound healing. A previous study reported that Smad4 inhibits wound healing by inhibiting keratinocyte proliferation. ${ }^{21}$ Another study also reported that dermatopontin has a profound role in wound healing by promoting keratinocyte proliferation. ${ }^{19}$ In our study, miR-124 overexpression significantly inhibited keratinocyte proliferation, while miR-124 silence markedly promoted keratinocyte proliferation, indicating that miR-124 might inhibit wound healing. The inhibitory effect of miR-124 on cell proliferation was also consistent with previous studies performed in cancer cells. ${ }^{22,23}$ To our knowledge, it is the first time the role of miR-124 in wound healing has been explored.

In order to close a wound opening, maturing ECM is recruited. ${ }^{24}$ As collagen is a major component of ECM, collagen biosynthesis is critical to both normal and pathological skin wound healing and can greatly affect the quality and outcome of healing. ${ }^{25}$ Zhou et al. demonstrated that the rate of collagen synthesis was increased during wound healing in muscle. ${ }^{26}$ Wang et al. also illustrated that calcium alginate accelerated the process of wound healing by improving type I collagen synthesis. ${ }^{27}$ Therefore, we also explored alterations of collagen biosynthesis in keratinocytes with ectopic miR-124 expression. Our results showed that collagen synthesis was markedly decreased by miR-124 overexpression, while it was increased by miR-124 silence, indicating the potential inhibitory effect of miR-124 on wound healing.
MicroRNAs usually function by binding to the 3'UTR of target genes, so we further explored the possible target gene of miR-124. TargetScan algorithms predicted more than 2,000 genes as possible target genes of miR-124. SERP1, also termed ribosome-associated membrane protein 4 (RAMP4), is implicated in the stabilization of newly synthesized membrane proteins and in N-linked glycosylation. ${ }^{28}$ Previous studies have reported that heat shock protein (Hsp47), which localizes in the endoplasmic reticulum, is a collagen-specific molecular chaperone, and that miR-29b reduces collagen biosynthesis by inhibiting Hsp47. ${ }^{29,30}$ We therefore hypothesized that SERP1 might be a target gene of miR-124. The subsequent luciferase assay and the changes in SERP1 expression induced by ectopic expression of miR-124 verified this hypothesis. Additionally, we also evaluated the cell viability, colony formation and collagen content in keratinocytes transfected with miR-124 alone or accompanied by SERP1 overexpression. The results once again verified that SERP-1 is a target gene of miR-124.

The Wnt/ $\beta$-catenin signaling pathway has been widely explored; it is involved not only in cell proliferation and the cell cycle, but also in wound healing. ${ }^{31,32}$ Moreover, the $\mathrm{Wnt} / \beta$-catenin pathway has been proven to regulate the proliferation and apoptosis of keratinocytes in psoriasis lesions. ${ }^{33}$ Hence, we studied the expressions of key kinases involved in the $\mathrm{Wnt} / \beta$-catenin signaling pathway. In the canonical Wnt/ $\beta$-catenin pathway, Wnt activates the "destruction complex", which is composed of axin, adenomatous polyposis coli (APC) and GSK-3 $\beta$, and prevents the phosphorylation and ubiquitination of $\beta$-catenin. ${ }^{34}$ When the $\beta$-catenin in cytoplasm is accumulated and translocates to the nucleus, $\beta$-catenin binds with TCF/LEF-1 transcription factors and then activates the transcription of downstream target genes, including cyclinB1, cyclinD1 and CDK2. ${ }^{35,36}$ In the present study, miR-124 obviously upregulated GSK-3 $\beta$ expression, suggesting upregulation of the destruction complex. As might be expected, the expression of $\beta$-catenin was downregulated, and downstream TCF-4 and LEF-1 were also markedly downregulated. As a consequence, cell cycle-associated proteins, including cyclin B1, cyclin D1 and CDK2, were all downregulated by miR-124. Hence, we came to the conclusion that miR-124 might affect keratinocyte proliferation and the cell cycle by deactivating the $\mathrm{Wnt} / \beta$-catenin pathway.

Finally,, we have shown that miR-124 significantly inhibited cell proliferation and collagen synthesis in keratinocytes. SERP1 is a target gene of miR-124 and its 
overexpression could attenuate the effects of miR-124 on keratinocytes. Moreover, miR-124 significantly deactivates the Wnt/ $\beta$-catenin pathway. These findings imply that miR-124 silence in wounds might be an effective strategy for healing and that miR-124 is a potential therapeutic target for wound healing.

\section{References}

1. Velnar T, Bailey T, Smrkolj V. The wound healing process: An overview of the cellular and molecular mechanisms. J Int Med Res. 2009;37(5): 1528-1542.

2. Gottrup F. A specialized wound-healing center concept: Importance of a multidisciplinary department structure and surgical treatment facilities in the treatment of chronic wounds. Am J Surg. 2004;187(5A): 38s-43s.

3. Gosain A, DiPietro LA. Aging and wound healing. World J Surg. 2004; 28(3):321-326.

4. Guo S, DiPietro LA. Factors affecting wound healing. J Dent Res. 2010; 89(3):219-229.

5. Takeo $\mathrm{M}$, Lee $\mathrm{W}$, Ito $\mathrm{M}$. Wound healing and skin regeneration. Cold Spring Har Perspect Med. 2015;5(1):a023267.

6. Huang C, Akaishi S, Hyakusoku H, Ogawa R. Are keloid and hypertrophic scar different forms of the same disorder? A fibroproliferative skin disorder hypothesis based on keloid findings. Int Wound J. 2014;11(5):517-522.

7. Ameres SL, Zamore PD. Diversifying microRNA sequence and function. Nat Rev Mol Cell Biol. 2013;14(8):475-488.

8. Banerjee J, Chan YC, Sen CK. MicroRNAs in skin and wound healing. Physiol Genomics. 2011;43(10):543-546.

9. Wang JM, Tao J, Chen DD, et al. MicroRNA miR-27b rescues bone marrow-derived angiogenic cell function and accelerates wound healing in type 2 diabetes mellitus. Arterioscler Thromb Vasc Biol. 2014; 34(1):99-109.

10. Wang $T$, Feng $Y$, Sun $H$, et al. miR-21 Regulates skin wound healing by targeting multiple aspects of the healing process. Am J Pathol. 2012;181(6):1911-1920.

11. Xie C, Shi K, Zhang X, Zhao J, Yu J. MiR-1908 promotes scar formation post-burn wound healing by suppressing Ski-mediated inflammation and fibroblast proliferation. Cell Tissue Res. 2016;366(2):1-10.

12. Zhang H, Wang $\mathrm{Q}$, Zhao Q, Di W. MiR-124 inhibits the migration and invasion of ovarian cancer cells by targeting SphK1. J Ovarian Res. 2013;6(1):84.

13. Zhenhua LI, Huang J, Zhenhong HU, Gao S. Effect of miR-124 on migration and invasion of non-small-cell lung cancer cells. Shandong Medical Journal. 2016;56:18-20.

14. Zhang YH, Wang QQ, Li H, Ye T, Gao F, Liu YC. miR-124 radiosensitizes human esophageal cancer cell TE-1 by targeting CDK4. Genet Mol Res. 2016;15(2). doi:10.4238/gmr.15027893

15. Li L, Luo J, Wang B, et al. MicroRNA-124 targets flotillin-1 to regulate proliferation and migration in breast cancer. Mol Cancer. 2013;12:163.

16. Walter MN, Wright KT, Fuller HR, MacNeil S, Johnson WE. Mesenchymal stem cell-conditioned medium accelerates skin wound healing: An in vitro study of fibroblast and keratinocyte scratch assays. Exp Cell Res. 2010;316(7):1271-1281.
17. Livak KJ, Schmittgen TD. Analysis of relative gene expression data using real-time quantitative PCR and the 2(-Delta Delta C(T)) Method. Methods. 2001;25(4):402-408.

18. Shibata S, Tada Y, Asano Y, et al. Adiponectin regulates cutaneous wound healing by promoting keratinocyte proliferation and migration via the ERK signaling pathway. J Immunol. 2012;189(6):3231-3241.

19. Krishnaswamy VR. Role of dermatopontin in re-epithelialization: Implications on keratinocyte migration and proliferation. Sci Rep. 2014;4:7385.

20. Santoro MM, Gaudino G. Cellular and molecular facets of keratinocyte reepithelization during wound healing. Exp Cell Res. 2005;304(1): 274-286.

21. Yang L, Li W, Wang S, et al. Smad4 disruption accelerates keratinocyte re-epithelialization in murine cutaneous wound repair. Histochem Cell Biol. 2012;138(4):573-582.

22. Sun Y, Ai X, Shen S, Lu S. NF-kB-mediated miR-124 suppresses metastasis of non-small-cell lung cancer by targeting MYO10. Oncotarget. 2015;6(10):8244.

23. Li X, Yu Z, Li Y, et al. The tumor suppressor miR-124 inhibits cell proliferation by targeting STAT3 and functions as a prognostic marker for postoperative NSCLC patients. Int J Oncol. 2015;46(2):798-808.

24. Kondo T, Ishida Y. Molecular pathology of wound healing. Forensic Sci Int. 2010;203(1-3):93-98.

25. Xue $M$, Jackson CJ. Extracellular matrix reorganization during wound healing and its impact on abnormal scarring. Adv Wound Care (New Rochelle). 2015;4(3):119-136.

26. Zhou S, Salisbury J, Preedy VR, Emery PW. Increased collagen synthesis rate during wound healing in muscle. PLoS One. 2013;8(3):e58324.

27. Wang T, Gu Q, Zhao J, et al. Calcium alginate enhances wound healing by up-regulating the ratio of collagen types $\mathrm{I} / \mathrm{III}$ in diabetic rats. Int J Clin Exp Pathol. 2015;8(6):6636-6645.

28. Pool MR. A trans-membrane segment inside the ribosome exit tunnel triggers RAMP4 recruitment to the Sec61p translocase. J Cell Biol. 2009;185(5):889-902.

29. Ito S, Nagata K. Biology of Hsp47 (Serpin H1), a collagen-specific molecular chaperone. Semin Cell Dev Biol. 2016;62:142-151.

30. Zhu Y, Li Z, Wang Y, et al. Overexpression of miR-29b reduces collagen biosynthesis by inhibiting heat shock protein 47 during skin wound healing. Trans/ Res. 2016;178:38-53.e36.

31. Yang HL, Tsai YC, Korivi M, Chang CT, Hseu YC. Lucidone promotes the cutaneous wound healing process via activation of the PI3K/AKT, Wnt/ß-catenin and NF-KB signaling pathways. Biochim Biophys Acta Mol Cell Res. 2016;1864(4):151-168.

32. Lu T, Bao Z, Wang $Y$, et al. Karyopherin $\beta 1$ regulates proliferation of human glioma cells via Wnt/ $\beta$-catenin pathway. Biochem Biophys Res Commun. 2016;478(3):1189-1197.

33. Zhang Y, Tu C, Zhang D, et al. Wnt/ $\beta$-catenin and Wnt5a/Ca2 ${ }^{+}$pathways regulate proliferation and apoptosis of keratinocytes in psoriasis lesions. Cell Physiol Biochem. 2015;36(5):1890-1902.

34. Klaus A, Birchmeier W. Wnt signalling and its impact on development and cancer. Nat Rev Cancer. 2008;8(5):387-398.

35. Jamieson C, Sharma M, Henderson BR. Targeting the $\beta$-catenin nuclear transport pathway in cancer. Semin Cancer Biol. 2014;27:20-29.

36. Ashihara E, Takada T, Maekawa T. Targeting the canonical Wnt/ $\beta$ catenin pathway in hematological malignancies. Cancer Sci. 2015; 106(6):665-671. 